\title{
Percepción docente del rendimiento académico de los estudiantes universitarios de la USAC Guatemala que trabajan y estudian
}

Edwin Rolando García Caal

Universidad de San Carlos de Guatemala, (USAC).Guatemala; garciacaal@gmail.com

Recibido: 24 de febrero del 2015

Corregido: 14 de abril del 2015

Aceptado: 31 de julio del 2015

\section{Resumen}

Esta investigación exploró la percepción de los docentes en relación con el rendimiento académico de estudiantes universitarios que además son trabajadores asalariados. Los docentes entrevistados pertenecen a la Facultad de Ciencias Económicas, campus central de la Universidad de San Carlos de Guatemala (USAC). Según se encontró en la literatura, para algunos autores la combinación "trabajar y estudiar en la universidad" tiene un efecto positivo en el rendimiento académico, así lo explican Caballero (2006) y Lillydahl (1990); en tanto que otros autores concluyen que dicha relación es negativa; tal como lo plantean Tyler (2003) y Paul (1982). Para validar una de estas dos posiciones, metodológicamente se realizaron las entrevistas a los docentes, y posteriormente se efectuaron observaciones estructuradas para profundizar en el contexto de las variables señaladas en las entrevistas. La mayoría de los entrevistados respondió que un trabajo asalariado es una de las causas que influencian negativamente el rendimiento académico universitario. Sin embargo, refirieron que ese efecto va precedido de "una baja calidad" de la educación preuniversitaria y dificultades en el acceso al campus. Se propone contrarrestar dicho efecto ampliando la capacidad docente y como solución no académica mejorar el acceso físico al campus universitario; al mismo tiempo se descubrió la necesidad de lograr una mejor definición del rol del estudiante universitario, porque los docentes pretenden que la universidad se adapte a las capacidades de los estudiantes y no al contrario.

Palabras clave: Criterios del rendimiento académico, Factores del rendimiento académico, Educación de adultos, Rendimiento académico, Progreso estudiantil, Efectividad docente.

\author{
Abstract \\ Academic performance of university students \\ from usac guatemala who works and studies \\ in the teachers' opinion
}

This research explores the teacher's perception hold of university students that also have a job. The teachers interviewed belong to the Faculty of Economics Science, central campus of the "Universidad de San Carlos de Guatemala" (USAC). It was found in literature, that for some authors the combination of work and study in the university has a positive effect in the academic performance, Caballero (2006) y Lillydahl (1990); while another authors conclude this relationship is negative, Tyler (2003) y Paul (1982). To validate one of these positions, methodological interviews were given to teachers. Subsequently structured observations were made to deepen the context of mentioned variables in the interviews. Most respondents replied that the fact of working is one of the causes that negatively influence in the university academic performance. However, teachers reported that this effect is preceded by "poor quality" of pre-university education and difficulties in accessing the campus. To counter this effect, it is proposed to use the teaching skills and as non-academic solution, improve physical access to campus; at the same time it was discovered the necessity to achieve a better defined university student role, because teachers claim that the university should meet the student capabilities and not the way around.

Key words: Performance criteria, Performance factors, Adult education, Academic achievement, Student progress, Teacher effectiveness. 


\section{INTRODUCCIÓN}

En el año 2011 se publicó en la página web de la Universidad de San Carlos de Guatemala un listado de 1215 estudiantes expulsados de las carreras de la Facultad de Ciencias Económicas (USAC, 2011). Dicha expulsión obedece a que según el Reglamento general de evaluación académica de dicha Universidad, aprobado por el Consejo Superior Universitario en el año 2005, cada estudiante tiene únicamente tres oportunidades de cursar una materia (USAC, 2005).

La medida administrativa se tomó al observarse que los estudiantes con bajo rendimiento académico congestionaban las aulas universitarias, dificultando las condiciones de aprendizaje de estudiantes con alto rendimiento académico; al mismo tiempo limitaban la oportunidad de ingreso a nuevos estudiantes y sobre todo limitaban los recursos financieros con los que la Universidad realiza sus acciones. Con esta medida se visualizaba un incremento en la promoción de los estudiantes universitarios, lo que traería consigo una reducción en el rezago.

En el artículo 6 del Reglamento citado, se define alto rendimiento académico como "el punteo que permite, en el proceso de evaluación, la aprobación de la asignatura con la nota mínima de promoción establecida en este reglamento, condición que el estudiante debe completar para lograr su promoción o ascenso a un curso superior"; en ese sentido el bajo rendimiento académico señala que "el estudiante no alcanzó como mínimo la nota de promoción establecida en este reglamento" (USAC, 2005). La normativa entró en vigencia en el año 2007 pero los resultados no han sido los esperados. En la Facultad de Ciencias Económicas, por ejemplo, el listado de los primeros estudiantes expulsados (año 2010), se vio incrementado durante el año 2011 y siguió la misma tendencia en el año 2012.

Como reacción a las expulsiones del primer semestre del año 2012, un grupo de 1230 estudiantes expulsados decidió tomar las instalaciones universitarias como represalia por la expulsión, exigiendo la anulación del reglamento de evaluación. El campus central fue cerrado, y en apoyo a los inconformes las asociaciones de estudiantes del Centro Universitario de Medicina (CUM) y de otros centros de estudios universitarios también tomaron medidas de hecho (El Periodico, 2012).
Lo que inició como un problema de la Facultad de Ciencias Económicas, terminó como un problema que afectó las actividades académicas de la Universidad en su conjunto. El Consejo Superior Universitario acordó como medida de solución, otorgar a los estudiantes una cuarta oportunidad que se hizo efectiva entre los meses de agosto y noviembre de 2012; adicionalmente, los estudiantes que hubieran repetido un curso dos veces y alcanzaron una nota insatisfactoria en el primer examen parcial de la tercera oportunidad, podrían presentar una carta de retiro del curso, lo que les permitiría repetir el curso nuevamente hasta lograr una nota satisfactoria.

El efecto de esa medida tampoco tuvo los resultados esperados, ya que en noviembre de 2013 el listado de los estudiantes expulsados se incrementó en 453, cifra mayor a los expulsados en noviembre de 2012 (USAC, 2013). Algunos docentes y personal administrativo plantean que el problema del rendimiento académico es más un problema de responsabilidad estudiantil que institucional, aunque aceptan una porción de responsabilidad en el tema de la calidad docente (CC.EE., 2012). Tales conjeturas y opiniones poco profundas que respaldan las soluciones implementadas, especulan sobre los factores que subyacen bajo el problema y atribuyen al esfuerzo individual estudiantil la solución al mismo, sin embargo, en materia científica dichos factores a la fecha son desconocidos y por lo tanto el énfasis se ha hecho únicamente en los efectos pero no en la causa.

Mediante respaldo teórico, es posible afirmar que el bajo rendimiento académico universitario no es un problema exclusivo de la Facultad de Ciencias Económicas o de la USAC en particular, ni siquiera de Guatemala; el mismo ha sido denunciado ampliamente a nivel mundial (UNESCO, 2005), pero los esfuerzos por entenderlo no han logrado la claridad necesaria para solucionar el problema y por lo tanto permanece como un tema de actualidad.

En el caso que nos aborda, la especulación sobre las razones del bajo rendimiento académico de los estudiantes de Ciencias Económicas, influye directamente en la decadencia del aprendizaje, según manifiestan los egresados (FAHUSAC, 2014) y también influye en la aplicación de medidas docentes y administrativas que no han logrado reducir la expulsión anual de 10\% de los estudiantes que mantienen bajo rendimiento 
académico en un mismo curso. Tales especulaciones tampoco han logrado reducir una deserción generalizada que alcanza $50 \%$ anual de los alumnos inscritos (Registro y Estadística de la USAC, 2014).

Con los antecedentes señalados y una delimitación del problema, se planteó hacer esta investigación siguiendo el siguiente objetivo: "Analizar con el apoyo de información obtenida en observaciones estructuradas, la percepción de los docentes sobre las causas que influyen en el rendimiento académico de los estudiantes de la Facultad de Ciencias Económicas en la USAC, con énfasis en el rendimiento esperado de aquellos estudiantes que estudian y trabajan".

A partir del análisis de los resultados se buscó plantear posibles medidas de solución al bajo rendimiento académico históricamente manifestado. La pregunta de investigación que orientó la metodología fue la siguiente: ¿Cuál es la perspectiva de los docentes respecto de las causas que influencian el rendimiento académico del estudiante universitario que trabaja y a la vez estudia en la Facultad de Ciencias Económicas? Luego de la transcripción de las entrevistas, se procedió con la observación estructurada de las variables planteadas por los docentes y se finalizó con el análisis de los factores más señalados.

\section{METODOLOGÍA}

Se decidió aplicar las técnicas de investigación en dos líneas de acción, en primer lugar se planteó el desarrollo de entrevistas a docentes de la Facultad de Ciencias Económicas en sus respectivas divisiones organizativas (Escuela de Economía, Escuela de Administración de Empresas, Escuela de Auditoría y Área Común). En segundo lugar se planeó el desarrollo de observaciones estructuradas, específicamente de observación directa de los aspectos señalados en las entrevistas, lo que incluye observación interpretativa, observación especulativa y análisis de entorno.

Para López y Deslauriers (2011), citando a Grawitz (1984), la entrevista puede tener un polo máximo de libertad y profundidad hasta un polo mínimo, con cuatro grados intermedios, a saber: 1. La entrevista clínica (psicoanálisis o psicoterapia), 2. La entrevista profunda, 3. La entrevista de respuestas libres, 4. La entrevista centrada o "focused interview", 5. La entrevista de preguntas abiertas, y 6 . La entrevista de preguntas cerradas.
En esta investigación se decidió hacer una entrevista centrada, la cual tiene por objeto la atención en una experiencia. Su método es más estricto que el de la entrevista de respuestas libres, ya que el objetivo en este tipo de entrevistas es bastante preciso. El entrevistado es libre de responder lo que desee, pero dentro del marco de la pregunta hecha. Los pasos seguidos fueron:

- Elaboración de la guía de entrevista;

- $\quad$ selección de los entrevistados;

- evaluación rápida de las características del sitio de la entrevista;

- estrategia de verificación (uso de sistemas de grabación) y

- creación de un sistema de codificación en función de recomendaciones teóricas (López Estrada \& Deslauriers, 2011).

Durante el proceso de investigación primero se realizó la revisión teórica de los conceptos que orientan la forma de elaborar técnicamente una guía de entrevista. Segundo se elaboraron seis preguntas orientadoras de la investigación, con la intención de focalizar la necesidad de información que se puede obtener a partir de la percepción que los docentes tienen del objeto de estudio. Tercero, se evaluaron las preguntas y se decidió la selección de una pregunta de ambientación, una pregunta de referencia y cuatro preguntas de información, con el interés de lograr que las entrevistas ocuparan un corto tiempo según la disponibilidad de los docentes para responderlas. Cuarto, se procedió con la elaboración de la guía de entrevista definiendo específicamente sus partes: parte introductoria, objetivo de la entrevista, preguntas orientadoras y cierre. Quinto, se realizó una validación o aplicación piloto de la guía de entrevista. De acuerdo con las sugerencias se reformuló el planteamiento de las preguntas. Sexto, se elaboró la versión definitiva de la guía de entrevista.

En la planificación de las entrevistas se tomó en cuenta la estructura organizativa de la Facultad de Ciencias Económicas, jornadas vespertina y nocturna. En la selección de los sujetos de entrevista se planificó un docente representante de los cursos cuantitativos (como por ejemplo, matemática, estadística y econometría) y un docente representante de los cursos cualitativos (por ejemplo, cursos del área social humanística o los cursos teóricos de especialización para la carrera) por cada uno de los ciclos de estudio establecidos en el currículo de las tres carreras que se imparten. 
Luego del consentimiento de los informantes, se procedió con la entrevista, dejando libertad a cada docente respecto del tiempo de duración de la misma. Cada pregunta se planteó de forma rápida y se facilitó una copia de la guía para incrementar el nivel de comprensión de las mismas. La información de referencia se solicitó hasta el final de la entrevista (nombre, salón, curso, edificio), la mayor parte de esta información se obtuvo por simple inspección.

Para la transcripción se procedió con la codificación de los audios. Posteriormente, se creó un sistema de categorías para la implementación del software de apoyo. Las respuestas fueron clasificadas por años de experiencia docente en la Facultad de Ciencias Económicas, con el fin de analizar posibles diferencias de percepción que se vieran influidas por esta variable.

\section{RESULTADOS}

La referencia al trabajo como causa del rendimiento académico universitario y el tema de la calidad de la educación preuniversitaria y su influencia en el rendimiento académico de los estudiantes de la Facultad, fueron los temas más abordados por los docentes. Cuando se les preguntó ¿cuáles considera usted que son las causas que influyen en el rendimiento académico del estudiante de nuestra facultad? Casi la totalidad respondió que el hecho de trabajar es una de las causas que lo influencian negativamente.

Lo anterior, se concibe desde la misma definición de estudiante universitario que hicieron los entrevistados. Utilizan como premisa que el estudiante de la Facultad es en primer lugar trabajador asalariado y luego estudiante y por lo tanto sus prioridades van en ese orden; explican que los estudiantes diurnos en una buena parte también trabajan. En las respuestas, no se encontró diferencia de opinión por la experiencia de los entrevistados. Tanto los docentes con más años de laborar en la Facultad de Ciencias Económicas como los de menor tiempo, expusieron que el trabajo es una limitante del rendimiento académico. A continuación se plantean algunas opiniones concluyentes.

Opinión 1. "Yo creo que una de las limitantes del éxito académico es que son estudiantes trabajadores, eso les limita poder dedicarse más a sus labores de estudiantes; vienen de 8 horas diarias de trabajo y pretenden continuar 4 horas adicionales poniendo atención a alguien que tal vez no da una clase amena, entonces pierden totalmente la atención; por lo tanto, un factor que está influyendo negativamente es ser estudiante-trabajador" (catedrático de curso cualitativo: experiencia entre 20 y 30 años).

Opinión 2. "El trabajo en la mayoría de los estudiantes que son de la jornada nocturna y fin de semana es absorbente; el tipo de trabajo que tienen depende de mucho tiempo" (catedrático de curso cuantitativo: experiencia entre 10 y 20 años).

Opinión 3. "El estudiante ya viene cansado, con sueño, y así no rinde igual, generalmente viene desvelado por salir de su casa en la madrugada; a ese desvelo se suma el cansancio por las 8 horas que trabajó durante el día" (catedrático de curso cuantitativo: experiencia 5-10 años).

Opinión 4. "Nuestra facultad se caracteriza porque la mayoría de estudiantes son trabajadores, eso influye mucho en su rendimiento académico; como trabajan no tienen el tiempo adecuado para dedicarle a los estudios" (catedrático de curso cualitativo: experiencia menor a 5 años).

Opinión 5. "Hay que adicionar el tema del transporte, este es un factor que hace que el estudiante no pueda venir a tiempo; viene a recibir los cursos ya cuando han empezado" (catedrático de curso cuantitativo: experiencia 5-10 años). Lo anterior va ligado a un pronunciamiento específico que un docente entrevistado hizo al tratar el tema del tráfico.

Opinión 6. "Tendría que hacerse un cambio en el pénsum de estudios; el estudiante universitario tendría que tener mejores condiciones para poder ingresar a la Universidad o tendrían que diversificarse los edificios en diferentes lugares de la ciudad capital para que el estudiante pudiera llegar más fácil “ (catedrático de curso cualitativo: experiencia 20-30 años).

Hasta este punto se señala que el trabajo (en las condiciones ya descritas) reduce el tiempo necesario para estudiar. Aunado a lo anterior, el tiempo de estudio también se ve reducido por el tema del retorno de la universidad hacia sus respectivos hogares. Esta idea se resume en los términos siguientes: 
Opinión 7. "El transportarse de un lugar a otro hace que el estudiante pierda mucho tiempo en ir a su casa, venir a la universidad y regresar de la universidad a su casa. Invierte demasiado tiempo en poco recorrido, en un día se tiene que transportar dos horas cuando vive cerca y cuatro horas de un lado a otro cuando vive lejos" (catedrático de curso cuantitativo: experiencia 1020 años).

Los entrevistados también señalaron que "la calidad de la educación preuniversitaria" puede modificar la influencia positiva o negativa del trabajo. Durante las entrevistas se descubrió que los años de experiencia como docentes universitarios no modifican tal percepción. En efecto, a pesar de las diferencias de experiencia docente, todos los entrevistados coinciden en que una de las principales causas del bajo rendimiento académico universitario de los estudiantes que trabajan es "la baja calidad de la educación preuniversitaria". Los comentarios señalan una influencia positiva cuando en la educación preuniversitaria ha existido buena calidad y lo contrario cuando el proceso se manifiesta débil. El planteamiento es el siguiente:

Opinión 8. "Lo mal preparado que el estudiante viene del nivel medio es una limitante cuando además de estudiar tiene que trabajar por un salario; viene sin las competencias y conocimientos necesarios para poder enfrentar el nivel de dificultad que se presenta en la universidad. Además de que la formación preuniversitaria es muy mala, la universidad no ha sido capaz de crear un espacio intermedio de preparación del estudiante al inicio de la carrera, para que comience a hacer un proceso de aprendizaje universitario de mayor nivel y de mayor exigencia. La universidad exige dedicarse a estudiar algo diferente a lo que aprendió en la primaria, en básicos y en diversificado. La consecuencia de una deficiente educación preuniversitaria, es que hay estudiantes con un bajo nivel académico y un bajo nivel de estudio; eso no les permite desarrollarse más allá de donde deberían" (catedrático curso cualitativo: experiencia 10-20 años).

Opinión 9. "Desde la parte del desarrollo académico intelectual que traen los estudiantes antes de su ingreso a la universidad, hasta la manera en la cual nosotros los recibimos y tratamos de elevarlos al nivel del conocimiento universitario, necesitan un apropiado seguimiento; sin embargo, no hay cursos que les capaciten para estar dentro de la universidad. La mala preparación se percibe a partir de que muchos candidatos pierden las pruebas específicas de ingreso (contabilidad, matemática, reglas de administración etc.), que son elementales para las ciencias económicas, entonces, si hubiera más seguimiento a todos los estudiantes, como por ejemplo, un curso preuniversitario en donde se establezcan todas esas características idóneas para poder pertenecer a una Facultad de Ciencias Económicas, estaríamos influyendo en las causas del bajo rendimiento "académico" y en el bajo rendimiento del estudiante en general" (catedrático de curso cualitativo: experiencia menor a 5 años).

Los docentes plantearon que no era su intención ratificar la mala preparación de los estudiantes en el nivel preuniversitario, sino señalar que a pesar de ser la principal razón del bajo rendimiento de los estudiantes asalariados, la Universidad no ha actuado en lo concerniente a la recepción de esos estudiantes. La mala calidad de la educación preuniversitaria no sólo influye en un bajo nivel académico (reprobar un curso), sino en un bajo nivel de estudio, entendido este último como el conjunto de competencias que hacen posible tener auto preparación. No es que los estudiantes no tengan conocimientos, es que no saben cómo adquirir esos conocimientos.

\section{DISCUSIÓN DE RESULTADOS}

En opinión de los docentes, el trabajador asalariado tiene desventaja cuando al mismo tiempo es estudiante universitario; no obstante, su desventaja puede variar en función de la calidad de la educación preuniversitaria. Si ha tenido una buena calidad en la educación preuniversitaria, la limitante laboral se vería compensada con una clase amena. En las entrevistas, se descubrió que lo señalado de negativo en las actividades laborales tiene que ver con el cansancio y la capacidad de poner atención en esas condiciones.

Se evidencia entonces que lo importante de la actividad docente debería centrarse en lograr que los estudiantes mantengan la atención en las clases y no en la transmisión de contenidos. Si la jornada laboral influye negativamente, "la extensión de la jornada laboral" influye aún más; aquí se está señalando la imposibilidad de los estudiantes para llegar temprano a recibir las 
clases y, en condiciones extremas, señala la imposibilidad de asistir a clases. Estos resultados coinciden con las hipótesis señaladas por Bordiu, citado por González y Pérez (2008, pág. 330), en donde específicamente se señala que:

1. Los estudiantes que dedican más horas a hacer sus deberes repiten menos.

2. Los estudiantes que suelen llegar tarde a clases son más propensos a repetir los cursos.

El planteamiento desde la percepción de los docentes confirma la necesidad de promover acciones de auto preparación y guías de trabajo para el aprendizaje extra-aula (tema que podría abordarse desde un entorno virtual).

La asistencia en opinión de los docentes es un factor que influye en el rendimiento académico. No obstante, señalan que las inasistencias son una consecuencia de las características del estudiante trabajador de Ciencias Económicas. En estas carreras la mayoría de estudiantes tienen título de perito contador (en la enseñanza secundaria). Los contadores deben realizar cierres contables cada mes e inventarios en las empresas; en ambos casos la inasistencia a clases es obligatoria.

En la literatura se señala que la ausencia a clases es una de las causas personales que incrementa la dificultad para adaptarse a la universidad, aunque la relación se hace con la falta de empatía con el profesor (González \& Daza, 2010). Este autor señala que la familia y las instituciones le exigen al estudiante universitario un comportamiento dual y contradictorio: por una parte, debe convertirse instantáneamente en adulto responsable asumiendo las responsabilidades del trabajo. Por otra, no aceptan la adultez en su comportamiento al no entregar tareas o asistir a clases, castigándolo con puntos por asistencia o por laboratorios hechos en clase, para lo cual debe seguir siendo adolescente obediente (González \& Daza, 2010). Esta forma de castigar las inasistencias con la pérdida de puntos, tiene una fuerte influencia en el bajo rendimiento académico. Por supuesto, la inasistencia no es una variable con influencia independiente.

Tejedor y otros (1998), mostraron que el alto rendimiento académico estaba influenciado en $65 \%$ por las variables siguientes: alumnos con alto rendimiento en la enseñanza secundaria, pertenecientes a los cursos del primer año de la carrera, con alta valoración en sus hábitos de estudio, que tienen un nivel bajo de inasistencias, con un nivel alto de satisfacción ante la carrera elegida, motivados culturalmente desde el ámbito familiar, con una actitud positiva hacia la Universidad y con un concepto de auto-eficacia elevado (Tejedor, y otros, 1998).

La investigación que nos aborda muestra también coincidencia en el tema de la alta valoración de los hábitos de estudio, lo que algunos docentes plantean como la alternativa a las inasistencias. Sin embargo, contrario a lo planteado en la investigación de Tejedor, la inasistencia en la USAC, Facultad de Ciencias Económicas, no influencia únicamente los resultados en los cursos del primer año de la carrera, ya que los docentes de semestres superiores también plantean la inasistencia por causas laborales como una limitante del rendimiento.

Otro aspecto importante por considerar se relaciona con el análisis de las condiciones contextuales de la asistencia y la inasistencia. Garbanzo (2007, pág. 61) realizó una sistematización de los factores asociados al rendimiento académico en múltiples investigaciones y advirtió que, dada la complejidad del tema, es fácil dejar fuera valiosos indicadores, en ese sentido concluye que no es posible generalizar sobre los factores, ya que los resultados pueden ser muy diferentes según las relaciones causales, las poblaciones y sus contextos. En este caso particular de la Universidad de San Carlos, el contexto laboral implica relaciones adicionales no encontradas en otras investigaciones.

Los docentes señalan que adicional a la jornada de trabajo se debe tomar en cuenta el tiempo para trasladarse hacia los centros de trabajo, lo que obliga a los estudiantes a salir de sus hogares de madrugada. Esto agrega la categoría "desvelo" al tema del cansancio. Sin embargo, el tema laboral es más complejo, porque además de la jornada de trabajo, en el caso de la USAC, debe considerarse la complicación del traslado hacia la universidad, tal como lo expresan los entrevistados.

Por otra parte, cuando se refirieron a los estudiantes de la jornada nocturna, hablaron de dos condiciones ligadas. Explicaron que llegar puntual a clases es una situación que no depende de los estudiantes sino de los problemas del transporte. De acuerdo con las observaciones realizadas, la jornada de trabajo finaliza media hora antes del inicio de las actividades estudiantiles universitarias, pero el tiempo de traslado hacia la Facultad no dura media hora. Se estableció que los 
buses no llegan al campus de la Universidad, sino que dejan a los pasajeros a distancias de 1, 2 y 3 kilómetros, según la ubicación de los edificios asignados a la Facultad de Ciencias Económicas. El tiempo de traslado de la "parada de bus" hacia las aulas varía de 30 a 45 minutos. Lo que significa que llegar puntual necesitaría una ubicación de los lugares de trabajo en los alrededores de la universidad, lo que no es posible para la mayoría de estudiantes.

El congestionamiento vehicular en la ciudad capital de Guatemala es un tema complicado y el campus universitario se encuentra ubicado entre dos avenidas que conducen a ciudades dormitorio (García, 2014). Lo expuesto significa que aún los estudiantes que poseen vehículo encuentran difícil su traslado del lugar de trabajo hacia el campus universitario en menos de $30 \mathrm{mi}-$ nutos. Adicionalmente, debe considerarse el tema de la saturación vehicular en el campus. Entrar a la USAC en carro es mucho más difícil que hacerlo a pie (factor acceso). Cuando cada semestre inicia, el ingreso al campus puede tardar entre 45 minutos y 1 hora con 15 minutos. Los parqueos son escasos (García, 2014). Tales inconvenientes no afectan a los estudiantes sin trabajo asalariado pero los inconvenientes a la salida sí. La complicación que los estudiantes encuentran al retornar a sus respectivos hogares también tiene muchas aristas. Para los estudiantes que tienen vehículo, salir del campus puede tardar hasta 60 minutos. Esta situación causa estrés y por supuesto reduce las ganas de llegar a estudiar a su casa, lo que es coincidente con las conclusiones de algunos autores respecto de la motivación para el estudio (Valle Arias, González Cabanach, Núñez Pérez, Martínez Rodríguez, \& Pineñor Aguin, 1999).

Como consecuencia de la situación descrita, un estudiante desmotivado para estudiar en su casa, tiende a mostrar un bajo rendimiento académico. Está claro que las variables espacial y temporal para el traslado de y hacia la universidad plantean efectos que han sido olvidados en la mayoría de investigaciones sobre el rendimiento académico. En ese sentido, es posible realizar una adaptación de la metodología de los efectos olvidados en el análisis, a partir de lo que se ha aplicado empresarialmente en la determinación de la rentabilidad de los efectos olvidados (Kaufmann \& Gil Aluja, 1988).

En el análisis del contexto que influye en la asistencia y la puntualidad de los estudiantes de la USAC, cobra importancia la Norma Municipal de la Ciudad Capital de Guatemala, que restringe la circulación de transporte pesado en horas pico (Acuerdo Municipal COM-005-2007), a este tipo de transporte se le permite iniciar a circular exactamente a la hora en que los estudiantes universitarios deben salir del campus.

El efecto de la normativa complica el retorno a sus hogares tanto a los estudiantes que ingresan con vehículo propio, como a quienes se movilizan en el transporte público. Como medida de solución, los estudiantes optan por salir de sus salones de clase entre 45 y 30 minutos antes de que finalice el periodo asignado a la última materia (García, 2014), por lo que además de llegar tarde a la primera clase por las anteriores causas, esta normativa les obliga a salir antes de que finalicen las clases. La dinámica que se analiza indica estrés y ansiedad. Al respecto se ha señalado que altos niveles de ansiedad pueden conducir a errores intelectuales, debido al compromiso en los procesos de memoria y dificultad de concentración de los estudiantes (Rains, 2004).

Se pudo establecer que los efectos señalados reducen directamente la jornada de clases. Los estudiantes, sin ser responsables de esto, no pueden terminar de recibir sus clases. Llegar tarde y salir temprano es una consecuencia del tema del transporte. Resumiendo el contexto, la jornada laboral y de estudio, en conjunto, ocupan un promedio de quince horas diarias de la vida de los estudiantes, situación que ejerce una presión muy alta sobre el rendimiento académico.

El hallazgo citado concuerda con la relación entre presión temporal y rendimiento descrita por la Ley de Yerkes-Dodson (1908), en la que la actuación óptima se produce en los valores medios de presión temporal (Freedman y Edwards, 1988; Pérez y Garrido, 1994). En otras palabras, tendrán un bajo rendimiento académico aquellos alumnos que tengan demasiado tiempo para el estudio y aquellos que no cuentan con el tiempo medio necesario. Esta relación ha recibido un importante soporte empírico por los resultados de diversas investigaciones (Isenberg, 1981; Karau y Kelly, 1992; Lanzetta, 1955 , entre otros), las cuales muestran que se consigue un desempeño óptimo cuando la presión temporal es moderada, mientras que dicho desempeño es menor cuando la presión temporal es muy alta o muy baja (Cladellas \& Castelló, 2011). La situación deja el rendimiento académico de los estudiantes a merced de sus capacidades de autoformación, pero tal capacidad depende directamente de la calidad de su educación preuniversitaria. Una educación preuniversitaria de baja 
calidad condena a los estudiantes-trabajadores a tener un bajo rendimiento académico universitario.

La investigación también evidenció que la influencia desfavorable de la baja calidad de la educación preuniversitaria no tiene una tendencia que señale únicamente una mala preparación para los cursos de base matemática o para los cursos teóricos. Ambos grupos de docentes señalaron esa limitación en términos similares. La relación que se encuentra entre estas opiniones y la teoría podría consensuarse con lo que señala Javier Touron Figueroa de la Universidad de Navarra. Este autor explica que hay un conjunto de variables que atienden a los resultados académicos de los alumnos en el pasado inmediato. Existe en esta referencia una variable denominada rendimiento medio global (Touron, 1984). Lo expuesto señala que los estudiantes con un bajo nivel de aprendizaje en el pasado inmediato del nivel universitario, como consecuencia tendrán un bajo rendimiento en la universidad.

Lo mismo se ha investigado en Universidades de Estados Unidos, en donde se emplean variables denominadas GPA o UGPA (grade point average o undergraduate point average), que analizan los rendimientos universitarios previos (Touron, 2011). No obstante, la relación entre los rendimientos académicos universitarios y los rendimientos previos depende de una investigación de índole cuantitativa, necesaria para ampliar el estudio. El interés estaría en establecer el grado de correlación que existiría entre estas variables.

La baja calidad de la educación preuniversitaria influye en varios aspectos, porque el estudiante puede llegar a la universidad mal preparado, pero con las capacidades para auto prepararse en respuesta a las presiones de la educación universitaria y la presión laboral, o por lo menos con la intención de nivelarse. Pero si el estudiante tampoco tiene esa capacidad de autopreparación, el problema se agrava.

De acuerdo con una publicación del Instituto Científico de Investigaciones Aplicadas al Ambiente y Desarrollo (Iciaad, 2012, pág. 43), las razones de la baja calidad de la educación preuniversitaria en Guatemala se originan en las estrategias del Ministerio de Educación para lograr el cumplimiento de los Objetivos del Milenio a partir del año 2001. En la primaria se promovió la estrategia Salvemos Primer Grado a través de la cual se planteó la obligación de evitar que niños de primer grado reprobaran el ciclo académico; aunque la estrategia impulsaba horas adicionales de refuerzo, los infantes fueron promovidos al grado superior sin saber leer y escribir. En el año 2004, dicha estrategia fue ampliada a los seis grados de primaria, se obligó así a los docentes a hacer exámenes remediales en cada bimestre para que los estudiantes pudieran recuperarse de los exámenes perdidos. En lugar de hacer exámenes remediales, los docentes evitaron que los alumnos reprobaran el grado aunque no estuvieran aptos para el grado superior.

A partir de estas condiciones, un conjunto de variables (salario tardío, escuelas de un maestro para todos los grados, trabajo infantil, riesgo de la rentabilidad de los colegios privados, docentes en extrema pobreza y ley del menor esfuerzo) influyeron en que los estudiantes fueran promovidos a los grados superiores sin contar con las capacidades para aprender, tratando de cumplir sólo los contenidos planteadas en el currículo (Iciaad, 2012, pág. 44).

Los docentes entrevistados señalan que ante esa problemática, la universidad debe adecuarse al nivel de preparación que traen los estudiantes y no ellos actuar en función de las exigencias del nivel universitario, pero esta posición no es la correcta. Estas opiniones sólo evidencian la necesidad de capacitar a los estudiantes para entender su propia función de universitarios y su rol de autoformación y a los docentes para comprender cuál debe ser la posición de la universidad. Este concepto es llamado autoconcepto académico y su influencia positiva en el rendimiento académico universitario ha sido demostrada por investigadores de la Universidad de Valencia (Gargallo López, Garfella Esteban, Sánchez Peris, Ros Ros, \& Serra Carbonell, 2009).

En ese sentido, aunque los docentes opinaron que era urgente buscar estrategias para hacer más digerible, más fácil y más práctico estudiar en la universidad, se percibe una posición paternalista que influye en el nivel académico de los egresados. La propuesta sólo es aceptada si no reduce la cantidad, ni la calidad de los contenidos de formación universitaria. Sin embargo, si los docentes no apoyan el autoconcepto académico no podrá construirse, ya que se señala en la literatura que su construcción se logra en interacción con el medio. En esa vía, es necesario que tanto los estudiantes como los docentes comprendan que una baja calidad de la educación preuniversitaria no implica una adaptación de la universidad a la ausencia de competencias estudiantiles, pero sí exige intervenciones puntuales para 
lograr elevar dichas competencias estudiantiles antes de su ingreso a la universidad.

\section{CONCLUSIONES}

De acuerdo con la información analizada, es posible concluir que para los docentes la jornada laboral de los estudiantes universitarios sumada a la jornada de estudio, en el contexto planteado, influyen negativamente sobre el rendimiento académico de los estudiantes universitarios, en la línea de investigación de Tyler (2003) y Paul (1982), quienes refieren que a más horas de trabajo menor rendimiento académico.

Los docentes agregan que, debido a lo prolongado (en tiempo) que resulta el traslado residencia-trabajo, trabajo-universidad y universidad-residencia, el estudiante realiza su proceso de aprendizaje en condiciones de cansancio y con poca capacidad de poner atención. Este elemento sugiere la necesidad de buscar técnicas que equilibren esta debilidad en función de los objetivos académicos (solución académica), fundamentándose en capacidades especiales del cuerpo docente, pero sin olvidar que los estudiantes deben ser aptos para el aprendizaje universitario (nivelación previa) y capacidad de autoformación.

Es oportuno considerar que llegar tarde y salir temprano es una consecuencia de las condiciones no académicas que impiden la presencia del estudiante en las aulas, lo que limita obligatoriamente el tiempo necesario para el aprendizaje, pero esta limitante se puede reducir si se mejoran las estrategias de entrega de los contenidos, ya sea en forma presencial o virtual; sin embargo, en ningún momento debe considerarse una reducción de temas.

Las condiciones geográficas de ubicación de la Facultad de Ciencias Económicas y los problemas de transporte impiden al estudiante llegar temprano y permanecer en el aula el tiempo establecido en el currículo, y a menos que se modifiquen las condiciones de acceso (solución no académica) el rendimiento académico estará presionado hacia abajo. Mientras estas relaciones continúen existiendo, el tiempo de estudio en sus lugares de residencia continuará restringido, situación que no es posible cambiar.

Sobre el tema de la mala preparación de los estudiantes a nivel preuniversitario se descubre una debilidad adicional, los estudiantes no cuentan con las capacidades para auto prepararse y en materia de definición de roles puede ser que, influenciados por la percepción docente, consideren que es la universidad la que debe adecuarse a su nivel de preparación y no ellos actuar en función de las nuevas exigencias del nivel universitario, lo que exige asumir el reto de actuar en función de la autodefinición del rol del estudiante, tanto a nivel del propio estudiante como a nivel del cuerpo docente, quien se encuentra apadrinando la idea de una universidad paternalista.

Bajo estas condiciones, si el estudiante universitario es a la vez asalariado y ha tenido una baja calidad en la educación preuniversitaria, la percepción docente señala que se espera que tenga un bajo rendimiento académico, influencia negativa que se podría reducir si se logra una mejora en el acceso al campus universitario y si se elevan las capacidades docentes para mantener la atención del estudiantado y promover un aprendizaje efectivo.

\section{REFERENCIAS}

Antia, M., Lima, J., \& Castro, L. (1994). La universidad adolescente. Bogotá: Fondo resurgir FES.

Boado, M. (2004). Una aproximación a la deserción estudiantil universitaria en Uruguay. Instituto Internacional para la Educación Superior en ALAC, UNESCO/IESALC.

Caballero, C. (2006). Burnout, engagement y rendimiento académico entre estudiantes universitarios que trabajan y aquellos que no trabajan (16 ed., Vol. 9). Revista Psicogente. Obtenido de http://132.248.9.1:8991/hevila/ Psicogente/2006/vol9/no16/1.pdf

CC.EE. (2012). Informe de sesión y Acta 7-2012 de la Sesión Celebrada por la Junta Directiva. Guatemala: Facultad de Ciencias Económicas.

Cladellas, R., \& Castelló, A. (2011). Percepción de disponibilidad temporal: efectos sobre los resultados en evaluaciones de niños de sexto grado de primaria. Barcelona: Revista de Psicodidáctica 16(2) 291-310.

Deslauriers, J. P. (2011). La entrevista cualitativa como técnica para la investigación en Trabajo Social. Nuevo León, México: Universidad Autónoma de Nuevo León.

El Periodico. (26 de abril de 2012). Noticias Nacionales. Recuperado el 27 de mayo de 2012, de www.elperiodico. com.gt/es/20120426/pais/211314 
Escobar, V. (2005). Estudio sobre la deserción y repitencia en educación superior en Panamá. Panamá: IESALC/UNESCO.

FAHUSAC. (2014). Seguimiento a graduados de la Universidad de San Carlos de Guatemala (documento en proceso). Guatemala: Dirección General de Investigación, DIGI.

Garbanzo V., G. M. (2007). Factores asociados al rendimiento académico en estudiantes universitarios, una reflexión desde la calidad de la educación pública. redalyc, 31(1), 43-63.

García, E. (5 de Mayo de 2014). Observación estructura de las causas que influencian negativamente el rendimiento académico universitario. Informe de observación. Guatemala, Guatemala: Documento de trabajo.

Gargallo López, B., Garfella Esteban, P. R., Sánchez Peris, F., Ros Ros, C., \& Serra Carbonell, B. (2009). La influencia del autoconcepto en el Rendimiento Académico Universitario. España: Universidad de Valencia.

Gómez, D., Oviedo, R., \& Martínez, E. (2011). Factores que influyen en el rendimiento académico del estudiante universitario. San Luis Potosí, México: Tecnociencia.

González, J. J., \& Pérez, J. C. (2008). Los orígenes del fracaso escolar en España, un estudio empírico. Madrid: CAJAMAR.

González, L., \& Daza, D. (2010). Repitencia en estudiantes de medicina, caracterización y causas. Puerto Colombia: Próxima Zona.

Iciaad. (2012). Principales manifestaciones de los problemas socioeconómicos de Guatemala. (E. R. García C., Ed.) Guatemala: Departamento de Educación del Iciaad.

IESALC. (2010). La Universidad Latinoamericana en discusión. Caracas, Venezuela: IESALC/UNESCO.

Kaufmann, A., \& Gil Aluja, J. (1988). Modelos para la investigación de efectos olvidados. (Vigo, Ed.) Barcelona: Milladoiro.

Lillydahl, J. (1990). Academic achievement and part-time employment of High school students. (L. Taylor \& Francis, Ed.) Journal of Economic Education, 21(3), 307-316. Obtenido de http://www.jstor.org/stable/1182250

López Estrada, R. E., \& Deslauriers, J.-P. (junio de 2011). La entrevista cualitativa como técnica para la Investigación en Trabajo Social. Margen. Obtenido de http://www.margen.org/suscri/margen61/lopez.pdf

Passeron, P. B. (1967). Los estudiantes y la cultura. París: Nueva colección Labor, Les Editions Minuit.

Paul, H. (1982). The impact of outside employment on student academic achievement in Macroeconomic principles. Journal of Economic Education, 51-56.

Rains, D. (2004). Principios de neuropsicología humana. México: McGraw Hill.
Registro y Estadística de la USAC. (10 de Julio de 2014). Archivo electrónico y base de datos de los créditos académicos de los estudiantes. Guatemala, Guatemala.

Serbia, J. M. (2007). Diseño, muestreo y análisis en la investigación cualitativa. Buenos Aires, Argentina: Universidad Nacional Lomas de Zamora, Facultad de Ciencias Sociales.

Tejedor, F. J., Zorrilla, A., García-Valcarcel, Herrera García, M., Nieto, M., Rodríguez, C., \& Sánchez, G. (1998). Los alumnos de la Universidad de Salamanca. Características y rendimiento académico. Salamanca: Ediciones Universidad Salamanca.

Touron, J. (1984). Factores del rendimiento académico en la Universidad. Pamplona, Navarra, España: EUNSA.

Touron, J. (2011). La predicción del rendimiento académico: procedimientos, resultados e implicaciones. España: Universidad de Navarra.

Tyler, J. (Abril de 2003). Using state child labor laws to identify the effect of school-year work on High school achievement. (T. U. Press, Ed.) Journal of Labor Economics, 21, 381-408. Obtenido de http://www.jstor.org/ stable/10.1086/345562

UNESCO. (2005). Proyecto Regional de Deserción y Repitencia en la Educación Superior en América Latina y el Caribe. IESALC.

USAC. (2003). Plan Estratégico USAC 2022, aprobado por el Consejo Superior Universitario el 26/11/2003. Guatemala: Consejo Superior Universitario, USAC.

USAC. (21 de julio de 2005). USAC Universidad de San Carlos de Guatemala. (A. 3.-2. Consejo Superior Universitario, Editor) Recuperado el 27 de mayo de 2012, de Facultad de Ciencias Económicas, Normativo General de Evaluación y Promoción del estudiante universitario: http://www. usac.edu.gt/secundario_dua.php?c=2260\&f=cienciasec

USAC. (21 de Diciembre de 2011). USAC Universidad de San Carlos de Guatemala. Recuperado el 29 de mayo de 2012, de Facultad de Ciencias Económicas: http://www.usac.edu.gt/secundario_dua. php?c $=5590 \& \mathrm{f}=$ cienciasec

USAC. (14 de 11 de 2013). Usac Tricentenaria. Recuperado el 21 de 7 de 2014, de Facultad de Ciencias Económicas: http:// www.usac.gob.gt

Valle Arias, A., González Cabanach, R., Núñez Pérez, J., Martínez Rodríguez, S., \& Pineñor Aguin, I. (1999). Un modelo causal sobre los determinantes cognitivomotivacionales del rendimiento académico. Revista de Psicología General Aplicada(52 (4)), 499-519. 